University of Nebraska - Lincoln

DigitalCommons@University of Nebraska - Lincoln

Faculty Publications in Computer \& Electronics Electrical \& Computer Engineering, Department Engineering (to 2015)

9-2009

\title{
Pre-filtering of Self-Encoded Spread Spectrum in Dense Multipath Channels
}

Won Mee Jang

University of Nebraska-Lincoln, wjang1@unl.edu

Lim Nguyen

University of Nebraska-Lincoln, Inguyen1@unl.edu

Follow this and additional works at: https://digitalcommons.unl.edu/computerelectronicfacpub

Part of the Computer Engineering Commons

Jang, Won Mee and Nguyen, Lim, "Pre-filtering of Self-Encoded Spread Spectrum in Dense Multipath Channels" (2009). Faculty Publications in Computer \& Electronics Engineering (to 2015). 64.

https://digitalcommons.unl.edu/computerelectronicfacpub/64

This Article is brought to you for free and open access by the Electrical \& Computer Engineering, Department of at DigitalCommons@University of Nebraska - Lincoln. It has been accepted for inclusion in Faculty Publications in Computer \& Electronics Engineering (to 2015) by an authorized administrator of DigitalCommons@University of Nebraska - Lincoln. 


\title{
Pre-filtering of Self-Encoded Spread Spectrum in Dense Multipath Channels
}

\author{
Won Mee Jang and Lim Nguyen \\ The Peter Kiewit Institute of Information Science, Technology \& Engineering \\ Department of Computer and Electronics Engineering \\ University of Nebraska - Lincoln \\ Omaha, NE 68182 \\ wjang1@unl.edu,.nguyen1@unl.edu
}

\begin{abstract}
We present pre-filtering methods of self-encoded spread spectrum (SESS) in dense multipath channels. SESS eliminates the need for traditional transmit and receive pseudo noise $(\mathrm{PN})$ code generators. In this paper, we propose multipath management of SESS in dense multipath urban environments. We propose a transmitter pre-filtering method that retains only good paths that contribute to the data decision positively at the receiver, and eliminates undesirable paths. The proposed technique significantly improves the system performance while increasing the transmit power moderately.

Index Terms - multipath, pre-filter, self-encoded spread spectrum.
\end{abstract}

\section{INTRODUCTION}

Self-encoded spread spectrum (SESS) is a novel modulation technique that acquires its spreading sequence from the randomness of an input data stream rather than through the use of the traditional pseudo-random $(\mathrm{PN})$ code generator. As the term implies, the spreading code is obtained from the random digital information source itself [1]. The statistical properties of random codes offer a number of advantages over the deterministic PN codes. Figure 5 shows an example block diagram of SESS system where the current symbol of duration $\mathrm{T}$ is modulated at the chip rate of $\mathrm{N} / \mathrm{T}$ by $\mathrm{N}$ previous symbols that have been stored in the registers. The self-encoded spreading chip sequence of length $\mathrm{N}$ not only has been generated from the random symbols and is independent of current symbol, but also is changing dynamically from one symbol to next. At the receiver, the feedback demodulator performs the reverse operation for symbol recovery by means of a correlation detector. The differential encoder and decoder serve to mitigate the effect of error propagation [2].

Due to detection errors, the despreading sequence may not be exactly the same as the spreading sequence from the transmitter, causing self-interference (SI) at the correlator output and degrade the system performance at low SNR. SI can also magnify significantly in dense multipath channel environments. The multipath delay spread becomes an additional limitation on the system performance as higher data rates are increasingly required in wireless communications.

The benefit of synchronizing to the strongest multipath component has been presented in [3]. A validated multipath model in [4] provides the multipath frequency spectrum analysis that can be used to bound the frequency domain region for multipath mitigation. An adaptive filtering process is used for estimating the parameters of both direct and multipath signals. Multipath elimination is then performed by subtracting the estimated multipath effects from the measured correlation function [5]. These receiver-based techniques essentially seek to improve multipath performance by estimating the channel effects and compensating for them at the receiver. Yet another approach employs return-to-zero phase shift keying (PSK-RZ) as an anti-multipath modulation technique that has been shown to achieve a much improved bit error rate (BER) when the multipath delay difference is smaller than the symbol duration [6].

In this paper, we propose a pre-filtering technique that can be employed when the channel state information is available at the transmitter (via feedback or by means of time division duplex). Transmitter-based techniques are feasible when computational power is available, such as in a base station. Pre-filtering can then be applied in propagation environments that have a fixed number of channel impulse responses of a single cluster such as personal communication systems (PCS). Our proposed pre-filtering approach computes the effect of each signal path to determine whether it would affect the final data decision positively or negatively. The negative paths are eliminated through pre-filtering so that only positive paths are retained. We find that the pre-filtering method also increases the transmit power moderately. In our preliminary results, we employed random spreading sequences to implement self-encoded spread spectrum systems. Random spreading sequences provide an affordable analysis due to its statistical symmetry. Since differential encoding can reduce the effect of SI as shown in Figure 1, for simplicity our analysis assumes that SI is negligible.

\section{SySTEM MODEL}

In this paper, we consider a discrete number of independent multipath components such that received signal plus noise from a multipath channel with $K$ paths is given by

$$
r(t)=x(t)+n(t)
$$

where $n(t)$ represents an additive white Gaussian noise (AWGN) with a two-sided power spectral density $\sigma^{2}$. Here we 
consider base band signals. The received signal is specified by

$$
x(t)=A \sum_{i=-\infty}^{\infty} \sum_{k=1}^{K} \alpha_{k} s_{i}\left(t-\tau_{k}\right) b_{i} .
$$

The signature waveform, $s_{i}(t)$, is a binary random sequence defined in $i T \leq t<(i+1) T$ and consists of $N=T / T_{c}$ antipodal random binary chips where $T$ and $T_{c}$ are the symbol and chip durations, respectively. $A$ is the signal amplitude and $\alpha_{k}$ is the fading envelope of the $k$-th path. We assume perfect channel information of fading envelope and phase. $b_{i}$ represents the data bit of the $i$-th bit interval, and $b_{i} \in[-1,1]$. We use the binary phase-shift keying (BPSK) modulation to simplify the presentation. We assume that fading characteristics remain constant during the bit interval. The signature waveforms are taken to have unit energy, $\int_{i T}^{(i+1) T}\left|s_{i}(t)\right|^{2} d t=$ 1. At the transmitter, we apply pre-filter to manage multipaths. The correlation of each path is obtained as follows:

$$
f_{i}^{k}=\int_{i T+\tau_{k}}^{(i+1) T+\tau_{k}} x(t) s_{i}\left(t-\tau_{k}\right) d t .
$$

If $f_{i}^{k} b_{i} \geq 0$, we retain the path. Let $\Lambda$ be a set of such paths, i.e., $\Lambda=\left\{k: f_{i}^{k} b_{i} \geq 0\right\}$. Otherwise the path is eliminated using the pre-filter. Let the channel impulse response be, $h(t)=\sum_{k=1}^{K} \alpha_{k} \delta\left(t-\tau_{k}\right)$, or $H(\omega)=\sum_{k=1}^{K} \alpha_{k} e^{-j \omega \tau_{k}}$. Then, the pre-filter can be written as

$$
\Gamma(\omega)=\frac{\sum_{k \in \Lambda} \alpha_{k} e^{-j \omega \tau_{k}}}{\sum_{k=1}^{K} \alpha_{k} e^{-j \omega \tau_{k}}} .
$$

We can compute the power increase $(\beta)$ due to the pre-filter

$$
\beta=\frac{\int|\Gamma(\omega)|^{2} d \omega}{\int 1 d \omega},
$$

where $|\Gamma(\omega)|$ is the magnitude of the pre-filter. The integration is performed over the signal bandwidth. Therefore, we compare the pre-filter to the ideal low pass filter to compute the transmit power increase.

The output of the rake receiver matched to the $k$-th path during the $i$-th bit interval, is

$$
y_{i}^{k}=\alpha_{k} \int_{i T+\tau_{k}}^{(i+1) T+\tau_{k}} r(t) s_{i}\left(t-\tau_{k}\right) d t
$$

for the maximal ratio combine. The equal gain combine can be obtained with $\alpha_{k}=1$ in Eq. (6). Therefore,

$$
y_{i}^{k}=A\left(\alpha^{k}\right)^{2} b_{i}+I_{k}+\eta_{k},
$$

where $I_{k}$ is interference in the $k$-th finger due to other paths. $\eta_{k}$ is a Gaussian random variable with a variance equal to $\sigma^{2}$. The final decision on the $i$-th data bit is made based on $y_{i}=\sum_{k=1}^{K} y_{i}^{k}$, i.e., the estimated bit, $\hat{b}_{i}=\operatorname{sign}\left(y_{i}\right)$.

There is a closed form solution of the BER for a $K$-th order isolated multipath diversity [7]:

$$
P_{b}=\left[\frac{1}{2}(1-\mu)\right]_{k=0}^{K}\left(\begin{array}{c}
K-1+k \\
k
\end{array}\right)\left[\frac{1}{2}(1+\mu)\right]^{k}
$$

where

$$
\mu=\sqrt{\frac{\bar{\gamma}_{c}}{1+\bar{\gamma}_{c}}}
$$

and where $\bar{\gamma}_{c}$ is the average signal-to-noise ratio (SNR) per channel. In a spread spectrum system with random spreading sequences,

$$
\bar{\gamma}_{c}=\frac{E_{b} / N_{o}}{1+\rho \frac{(K-1)}{N} E_{b} / N_{o}}
$$

where we have incorporated the multipath interference term in the denominator for the Gaussian approximation. The interference is considered proportional to the number of interfering paths $(K-1)$ and inversely proportional to the spreading length $(\mathrm{N})$. The control factor, $\rho$, has been included in the equation to approximate the multipath interference accurately.

\section{Numerical Results}

For our simulation, we assume each path with Rayleigh fading and the delay is uniform within the bit time, i.e., $0 \leq \tau_{k} \leq T$. The delay is considered as a multiple of the chip duration, $T_{c}$. Figure 2 plots the performance of the equal gain combine for five paths and eight chips per bit. It can bee seen that multipath management using the pre-filter at the transmitter outperforms the without pre-filtering. Without pre-filtering, the performance approaches the BER floor of $10^{-3}$ at $E_{b} / N_{o}=12 \mathrm{~dB}$. Figure 3 shows a similar result for ten paths and 16 chips per bit, with the BER floor of $2 \times 10^{-5}$ at $E_{b} / N_{o}=12 \mathrm{~dB}$ without pre-filtering. In both figures, the proposed multipath management improves the BER significantly. Figure 4 shows the BER of the maximal ratio combine with five paths and eight chips per bit. The performance improvement with multipath management is also evident in the maximal ratio combine as expected. Figure 4 also plots the analytical BERs that have been computed from Eq. (8) for $\rho=0.13$ with pre-filtering, and for $\rho=0.7$ without pre-filtering. It can be seen that the effective multipath interference has been reduced about $18.5 \%$ by applying the pre-filter. Notice that the analytical BER is pessimistic at a high SNR due to the Gaussian approximation of the multipath interference. It has been shown that the Gaussian approximation of the synchronous random sequence interference tends to be pessimistic at a high SNR [8].

Table I shows the example power enhancement due to the pre-filter with 16 chips per bit and ten paths. The total number of transmissions was 598,630 bits at $E_{b} / N_{o}=0 \mathrm{~dB}$, although the SNR affects neither the pre-filter processing nor the corresponding power increase. From the simulation results, there was no path removal in 220,658 bits, i.e., all paths contribute to the final data decision positively for $36.86 \%$ of the total transmissions. The pre-filter removes seven paths and retain only three paths in 16 bits out of 598,630. That is, seven paths affect the final decision negatively, leaving only three positive paths for $0.003 \%$ of the total transmissions. The largest power increase factor is 5.9 , or $7.7 \mathrm{~dB}$, with five paths and the overall average power increase is 2.58 , or $4.1 \mathrm{~dB}$. We 
obtain a similar result from the simulation for five paths with eight chips per bit. In this case for 224,070 bits or $60.36 \%$ of the transmission the pre-filter does not suppress any paths. The overall average power increase is 1.74 or $2.4 \mathrm{~dB}$. However, Figure 4 displays more than a $2.4 \mathrm{~dB}$ performance enhancement. In fact, the error floor is observed as $10^{-4}$ and $10^{-7}$ with and without the pre-filter, respectively. The results demonstrate that pre-filtering can provide significant performance improvement with only a moderate power penalty. The proposed pre-filtering exhibits better performance than the minimum mean square error (MMSE) pre-filtering [9] or zero-forcing pre-equalizer [10] since our scheme retains only good paths selectively.

\section{CONCLUSIONS}

In this paper, we proposed a method to apply a correlative pre-filter at the transmitter to control multipath interference in random spread spectrum systems. Our analysis has shown that the system performance improved significantly with only a moderate increase in the transmit power. The proposed multipath management can be applied to the channel with a fixed number of impulse responses of a single cluster. Further work is underway to investigate the effect of self-interference in SESS on the proposed transmitter pre-filtering technique.

\section{ACKNOWLEDGMENTS}

This work was supported by the contract award FA9550-081-0393 from the U.S. Air Force Office of Scientific Research. Thanks are due to Dr. J. A. Sjogren whose support has allowed the authors to investigate self-encoded spread spectrum in dense multipath urban environments.

\section{REFERENCES}

[1] L. Nguyen, "Self-encoded spread spectrum and multiple access communications," Proceedings of the IEEE $6^{\text {th }}$ International Conference on Spread Spectrum Technology \& Applications, NJIT, NJ, Sept. 2000.

[2] Y. Kong, L. Nguyen and W. M. Jang, "Self-encoded spread spectrum modulation with differential encoding," IEEE International Symposium on Spread Spectrum Techniques and Applications, pp. 471-474, Sept. $2-5,2002$.

[3] P. Boyer, "Performance based on selective multipath reception," IEEE Trans. Commun., vol. 52, no. 2, pp. 280-288, Feb. 2004.

[4] Y. Zhang and C. Bartone, "Multipath mitigation in the frequency domain," IEEE Position Location and Navigation Symposium, PLANS 2004, pp. 486-495, 26-29 April 2004.

[5] M. Minami, H. Morikawa and T. Aoyama, "An adaptive multipath mitigation technique for GPS signal reception," IEEE Veh. Technol. Conf. Proc., VTC2000-Spring, vol. 2, pp. 1625-1620, 15-18 May 2000, Tokyo.

[6] S. Yoshida, T. Takeuchi, M. Nakamura and F. Ikegami, "High bit-rate field transmission of an anti-multipath modulation technique PSK-RZ," IEEE 40th Veh. Technol. Conf. Proc., pp. 527-532, 6-9 May 1990.

[7] J. G. Proakis, Digital Communications, $4^{\text {th }}$ ed., pp. 825 , McGraw Hill, 2001.

[8] W. M. Jang, L. Nguyen and P. Bidarkar, "MAI and ICI of synchronous downlink MC-CDMA with frequency offset," IEEE Trans. Wireless Commun., vol. 5, no. 3, pp. 693-703, Mar. 2006.

[9] L. Sanguinetti, M. Morelli and I. Cosovic, "MMSE pre-filtering techniques for TDD MC-CDMA downlink transmissions," 61st Veh. Technol. Conf., VTC 2005-Spring, vol. 3, pp. 1716-1720, 30 May - 1 Jun. 2005.

[10] H. Sampath, H. Bolcskei, A. J. Paulraj, "Pre-equalization for MIMO wireless channels with delay spread," 52nd Veh. Technol. Conf., VTS 2000-Fall, vol. 3, pp. 1175-1178, 24-28 Sept. 2000.

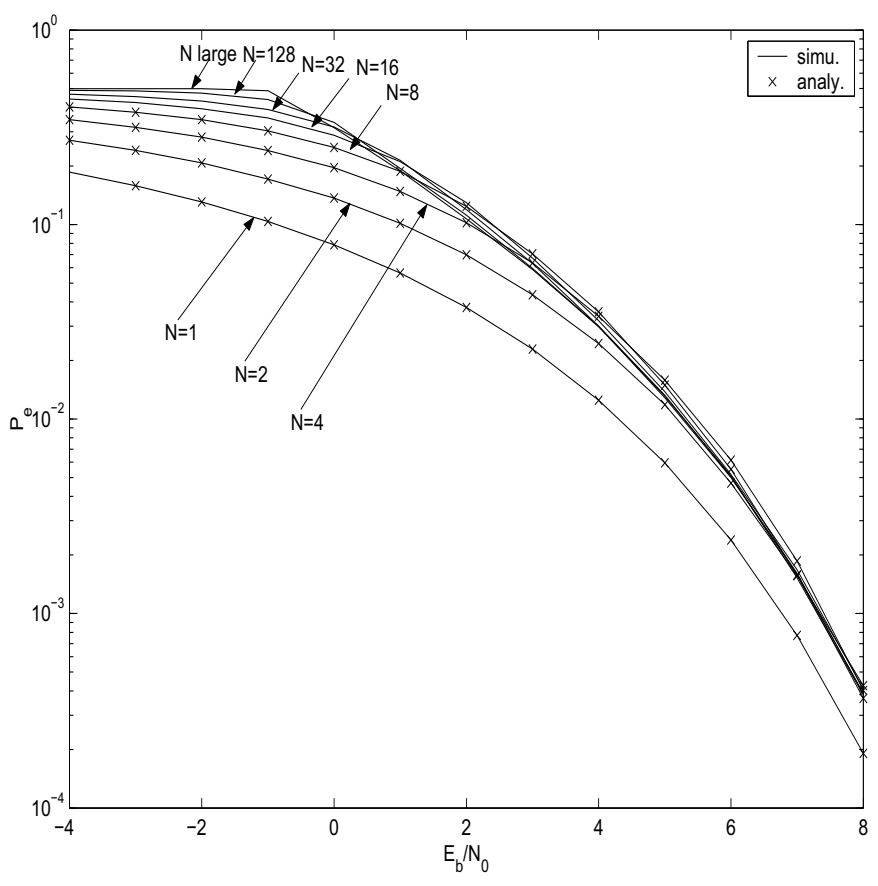

Fig. 1. BER of SESS with differential encoding where $\mathrm{N}$ is the spreading length.

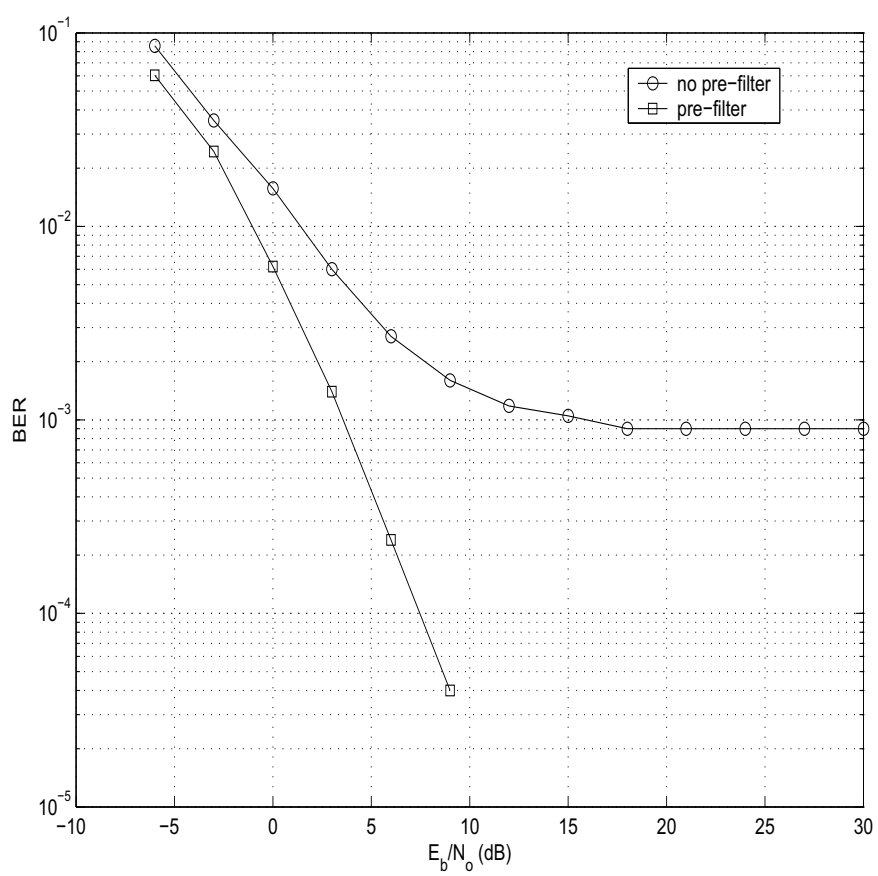

Fig. 2. Simulation BER, equal gain combine, $K=5, N=8$. 


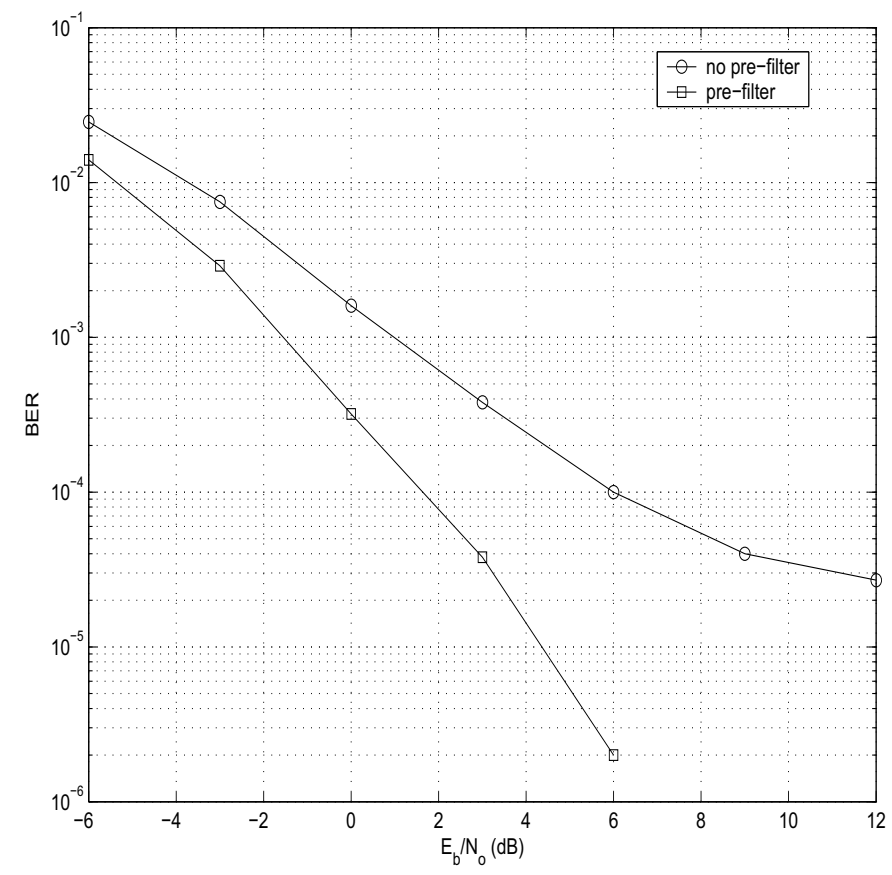

Fig. 3. Simulation BER, equal gain combine, $\mathrm{K}=10, \mathrm{~N}=16$.

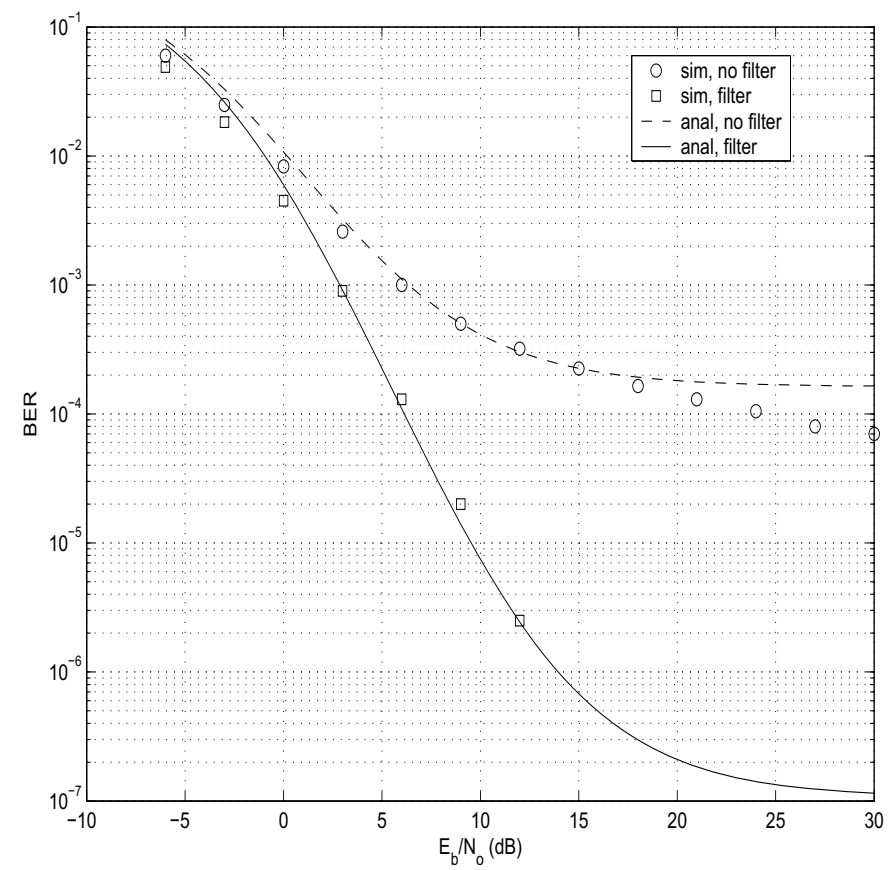

Fig. 4. BER, maximal ratio combine, $\mathrm{K}=5 \mathrm{~N}=8$. 
TABLE I

Transmit Power Increase, maximal Ratio combine, $\mathrm{K}=10$, N=16.

\begin{tabular}{|l|l|l|l|r|r|r|r|r|r|c|}
\hline Number of Paths Retained & 1 & 2 & 3 & 4 & 5 & 6 & 7 & 8 & 9 & 10 \\
\hline \hline Average Power Increase & 0 & 0 & 2.9 & 3.7 & 5.9 & 4.5 & 4.9 & 3.4 & 2.9 & 1.0 \\
\hline Percentage Transmission & 0 & 0 & 0.003 & 0.06 & 0.637 & 3.32 & 10.31 & 20.37 & 28.44 & 36.86 \\
\hline
\end{tabular}

Overall Average Power Increase: 2.58 (4.1 dB)

Total Number of Transmission: 598,630

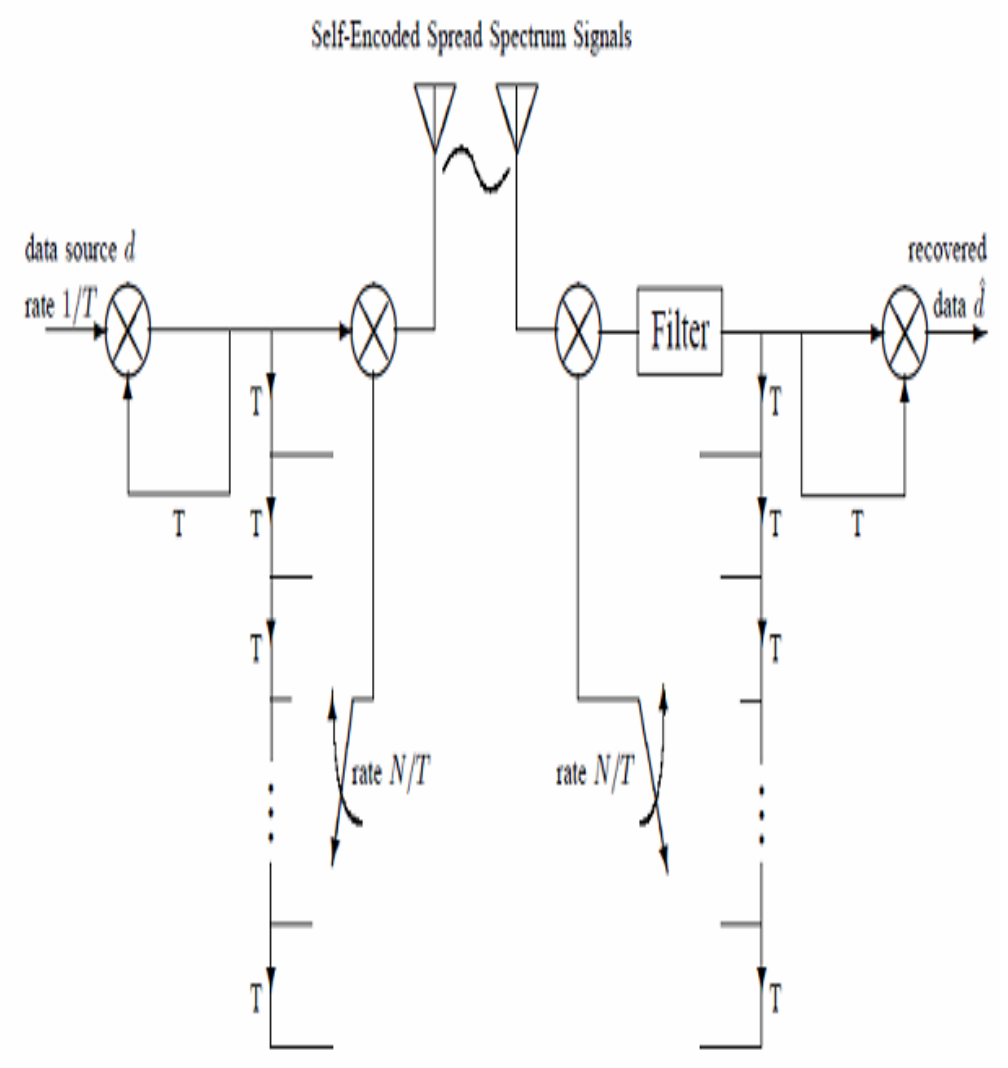

Fig. 5. Self-encoded, direct-sequence spread spectrum. 